\title{
PERFIL DE RIESGO EN DIABETES MELLITUS TIPO 2 DE UNA COMUNIDAD SEMIURBANA EN LA CIUDAD DE MÉXICO.
}

Mira. Verónica Flores Femández", Mtro. Liliang Gonzalez-Juárez*t y Mtra Gandhy Ponce Gómez"*."

"Profesor de Carrera Asociado "B". ENEO-UNAM, "Aprofesor de Carrera Tituiar "A". ENEO-UNAM, *wProfesor de Carrera Asociado "B". ENEO-UNAM

\section{RESUMEN}

La Encuesta Nacional de Enfermedades Crónicas (2000) en México, identificó un aumento en la prevalencia de diabetes mellitus tipo 2 del $7.2 \%$ a $10.9 \%$ en siete años, por lo tanto el propósito del estudio fue identificar los factores de riesgo para la diabetes mellitus tipo 2 de las personas de 20 a 69 años de edad de una comunidad suburbana de la ciudad de México, a fin de realizar un seguimiento longitudinal de los factores de riesgo de personas en estudio.

Se realizó una valoración casa por casa, hasta completar una $n=256$ personas del Área Geoestadística Básica (AGEB) 052-7 que se localizaron en el momento del estudio. El equipo de trabajo se sometió a un proceso de estandarización para realizar las valoraciones clinicas y aplicar la Cédula de Entrevista Familiar la cual incluye algunos datos tales como; glucosa capilar casual, indice de masa corporal, indice cintura cadera, entre otros, los parámetros de referencia fueron los establecidos por la Organización Mundial de la Salud (OMS), American Diabetes Association (ADA), Comité de Expertos sobre el Diagnóstico y Clasificación de la Diabetes Mellitus y el Comité de Unión Nacional de Prevención y Detección de la Hipertensión. El análisis estadístico se presenta en estadistica descriptiva se utilizó el paquete estadístico de SPSS versión 8.0.

Resultados.- El $12.5 \%$ refirió tener diagnóstico de diabetes y el promedio de años de diagnóstico fue de $7.6+$ 6.0 años. El $5.5 \%$ obtuvo cifras glucosa capilar casual de $\geq 200 \mathrm{mg} / \mathrm{dl}$. Se observó un incremento drástico de la glucosa capilar casual en los grupos de edad de 50 a 59 años y 60 a 69 años de edad. El Indice Cintura Cadera (ICC) de riesgo para el género femenino se encontró en el $68.5 \%>84$, para el género masculino el $47.2 \%$ se ubicó en $>.93$.

Es notorio que un gran porcentaje de la población en estudio muestra una tendencia a la obesidad y sobrepeso con elevadas cifras de IMC e ICC. Se desconoce la incidencia anual de la diabetes mellitus tipo 2, en población abierta, por lo que se sugiere realizar detecciones anuales.

PALABRAS CLAVES. Diabetes mellitus tipo 2, factores de riesgo.

\section{ABSTRACT.}

The National Chronic Diseases Survey (2000) in Mexico, identified a $7.2 \%$ to $10.9 \%$ increase in type 2 diabetes mellitus prevalence throughout seven years; the study purpose, therefore, vas to identify the type 2 diabetes mellitus risk factors in the 20 - 69 year old population within a Mexico City suburban community, aiming to perform a risk factor longitudinal follow-up with the people in the study.

A house-by-house assessment was carried out until a 052-7 Basic Geostatistical area (AGEB) with $n=-256$ people was completed; people was located at the moment of the study. The work team was subject to a standarizing process in order to perform clinical evaluations and applying the Family Interview Index which includes several data such as capillary glucose casual, body mass index, waist-hip index, among others, and the reference 
parameters were those established by the World Health Organization (WHO), the American Diabetes Association (ADA), the Experts Committee on Diabetes Mellitus Diagnoses and Classification, and the National Union Committee for Hypertension Prevention and Diagnoses. The statistical analysis is presented in a descriptive statistic and the SPSS version 6.0 statistical package was used.

The $12.5 \%$ of the sample score having a diabetes diagnoses, and the diagnoses media in years was $7.6+8.6$ years. $5.5 \%$ got casual capillary glucose figures of $\geq 200 \mathrm{mg} / \mathrm{dg}$. An awesome increase in casual capillary glucose within the 50-59 and 60-69 year old groups was observed. The ICC on risk for female was found on $68.5 \%>84$, and for males it was $47.2 \%$ located in $>93$.

It can be strongly noticed the a great percentage of the study sample shows a lean through obesity and over weight with high figures for IMC and ICC. The annual incidence for type 2 diabetes mellitus within the open population is unknown. Therefore, it is suggested to perform annual detection actions.

\section{KEY WORDS.}

Type 2 diabetes mellitus; risk factors; prevalence.

\section{INTRODUCCIÓN}

La Asociación Americana de Diabetes (ADA) estimó 35 millones de personas diabéticas a nivel mundial en el año 2000, de las cuales 19 millones vivían en América Latina y el Caribe. Las proyecciones indican que para el 2025 esta cifra ascenderá a 64 millones de los cuales 40 millones corresponderán a América Latina y el Caribe (1). Lo anterior se debe a factores tales como; la predisposición genética a la diabetes, incremento en la población de adultos y adultos mayores, cambios en los estilos de vida, sedentarismo, mayor consumo de carbohidratos y grasas saturadas entre otros.

La Encuesta Nacional de Salud (ENSA, 2000), registró una prevalencia de diabetes del 10.9\% y de obesidad $\left(<27 \mathrm{~kg} / \mathrm{m}^{2}\right)$ en el $48.8 \%$ entre la población mayor de 20 años. Por otro lado, refiere que el $62.5 \%$ de los pacientes con diabetes clasifican como obesos. Lo anterior indica la necesidad de implementar acciones que incidan en la prevalencia e incidencia del sobrepeso y obesidad.

Un factor relevante en algunos estudios es que la prevalencia de diabetes se incrementa gradualmente con la edad. La ENSA (2000), muestra que en el grupo de 20 a 39 años la prevalencia de diabetes mellitus tipo 2 fue de $2.3 \%$ y se incrementó severamente al $10.0 \%$ en la población de 40 a 49 años y este valor se duplica al $22.3 \%$ en el grupo de edad de 60 a 69 años. ${ }^{(2)}$
Vázquẹ Ch. C. et: al (2003) en un estudio sobre factores metabólicos de riesgo cardiovascular en población mexicana con diferente Indice de Masa Corporal (IMC), refieren que a mayor IMC se incrementa el Índice Cintura Cadera (ICC) y la tensión arterial indicando una predominancia de obesidad de tipo central en la población estudiada, siendo estos resultados estadísticamente significativos. Los niveles de glucosa mostraron incremento al aumentarse el IMC. is

Sánchez R. y col. (2001) utilizaron la base de datos de los pacientes que habían participado en los años 93-94 y 96-97, para determinar el número de nuevos casos con IMC $\geq 30,25-26.99$ y $27-29.99 \mathrm{~kg} / \mathrm{m}^{(4)}$. Se empleó el método de regresión logística para determinar los factores que influyen en la aparición de nuevos casos de obesidad y sobrepeso. Se siguieron 378 varones y 1,057 mujeres. Las conclusiones del estudio mostraron 10 siguiente; 1) La incidencia de obesidad en la población estudiada fue de $1.8 \%$ en hombres y de $6.7 \%$ en mujeres entre 20 y 65 años de edad. 2) La incidencia de obesidad y sobrepeso es mayor en mujeres que en hombres. 3) Tener el nivel IMC previo es el principal predictor del riesgo de obesidad y sobrepeso.

Como se observa los factores de riesgo para diabetes mellitus tipo 2 de la población mexicana han ido en ascenso principalmente en áreas urbanas, debido tal vez 
a los cambios en la alimentación, sedentarismo, escasa práctica de ejercicio asi como por el incremento de la población mayor de 20 años. Por lo tanto, el propósito de este estudio fue identificar los factores de riesgo para la diabetes mellitus tipo 2 de las personas de 20 a 69 años de edad de una comunidad suburbana de la ciudad de México, a fin de realizar posterior a este estudio una intervención y seguimiento longitudinal de los factores de riesgo de personas participantes.

\section{METODOLOGÍA}

El tipo de estudio fue descriptivo y transversal, ya que sólo se realizó una caracterización de la población en estudio durante los meses de junio a agosto de 2002. Se realizaron visitas casa por casa en un área de la comunidad suburbana de la delegación Xochimilco de la ciudad de México, se realizó la valoración de riesgos de la población de 20 a 69 años. Se conformó una muestra aleatoria no probabilistica de $n=256$ sujetos.

Previo al estudio se sometió al equipo de trabajo al proceso de estandarización para la entrevista y la toma de las mediciones de talla, peso, perimetros cintura-cadera, glucosa capilar y tensión arterial.

Como criterios de inclusión se encuentran las personas de 20 a 69 años de edad de ambos sexos que aceptaran participar libremente. Los criterios de exclusión descartaron a las personas con enfermedades infecciosas, cardiovasculares, gastrointestinales, renales y diabéticos tipo 1 . Así como a las personas con discapacidad física para comunicarse y movilizarse o indisposición para dar información bajo consentimiento y mujeres embarazadas. Como criterio de eliminación se consideraron a las personas con los datos de las cédulas incompletas.

La Cédula de Entrevista Familiar (CEF) fue estructurada por los autores del estudio y estaba conformada por dos apartados 1) con los datos de referencia de la familia, domicilio, parentesco, sexo, edad, ocupación, empleo, escolaridad, antecedentes de diabetes en primer grado (padres y hermanos) con diagnóstico previo y productos macrosómicos y el 2) incluía talla, peso, perímetro de cintura, perímetro de cadera, tensión arterial y glucosa capilar casual.

A la población que participó en el estudio, se le explicó el propósito del mismo y las condiciones en las que se realizarian las mediciones, a fin de que decidieran su participación. Para realizar las mediciones se les pidió a los participantes quitarse los zapatos, el exceso de ropa, joyas y colocarse una bata; pidiéndoles vaciamiento intestinal y vesical, con el objeto de obtener un dato más confiable, en las cifras de peso y los perimetros de cintura y cadera. Además de mantenerse así durante el desarrollo de todas las mediciones,

Una vez obtenidos los datos y realizadas las mediciones se procedió al registro de los mismos en la CEF: A la familia se le entregó un reporte que incluía un informe sobre su estado de salud, con base en los parámetros evaluados y con las recomendaciones para el cuidado de los problemas detectados. El formato del reporte fue codificado en base a los colores del semáforo, de acuerdo al color, el riesgo.

A los sujetos que no se localizaron en el momento de realizar las valoraciones, se planeó una segunda visita en caso de no estar presentes se decidió no incluirlos en el estudio,

Las diversas mediciones como: la estandarización de la glucosa capilar, peso, talla, tensión arterial y circunferencia de cintura-cadera, se detallan en el manual de procedimientos del proyecto "Estilos de Vida en Diabetes Mellitus tipo 2". (ESVIDIM-2, 2000) (s), el cual considera como parámetros de referencia los establecidos por la Organización Mundial de la Salud (OMS), The Expert: Comité on the Diagnosis and Classification of Diabetes Mellitus, Comité de Expertos sobre el Diagnóstico ${ }^{6}$ y Clasificación de la Diabetes Mellitus y el Comité de Unión Nacional de Prevención y Detección de la Hipertensión ?

La toma de glucosa capilar casual se llevó a cabo por medio de un glucómetro digital modelo One Touch Basic Plus marca Jhonson \& Jonson. El diagnóstico de diabetes mellitus se estableció bajo los criterios del Comité de Expertos sobre el Diagnóstico y Clasificación de la Diabetes Mellitus los cuales consideran como diabéticos a las personas con sintomas de diabetes mellitus, además de un registro de glucosa en plasma en ayuno $\geq$ $126 \mathrm{mg} / \mathrm{dl}$ en más de una ocasión o una glucosa en plasma casual $\geq 200 \mathrm{mg} / \mathrm{dl} 6$.

El peso se registró con una báscula digital de precisión, modelo Ultimate Scale , marca Tanita, con rango de 0 a 130 kilogramos. La talla con un altímetro metálico marca Torino plegable con escala de 0 a 2.10 metros.

La clasificación del IMC, se basó en los criterios establecidos por la Organización Mundial de la Salud?, el cual considera como referencia de clasificación el índice de Quetelet, que es la relación de peso corporal en kilogramos, dividido entre la estatura en metros al cuadrado el 
cual define Obesidad. Para la toma de tensión arterial se utilizó un monitor digital de presión sanguínea marca Omrom, modelo HEM-73.1C, en posición sedente y en condiciones de reposo.

Para el cálculo del Indice Cintura Cadera (ICC), se utilizó una cinta métrica flexible de 0 a $150 \mathrm{~cm}$, en posición anatómica. Se consideró como riesgo una relación cintura-cadera mayor de 0.93 para hombres y mayor de 0.84 en la mujer.

El análisis estadistico se presenta en tablas de frecuencia y porcentajes, medidas de tendencia central. La comparación entre dos grupos categóricos se realizó mediante X? Se utilizó el Paquete Estadístico para Ciencias Sociales (SPSS), versión 8.0 .

\section{RESULTADOS}

La muestra estuvo conformada por 256 personas de los cuales el $72 \%$ fueron mujeres y el $28 \%$ hombres. La edad de los sujetos fue de 20 a 69 años con un promedio de edad para las mujeres de 30.0 años + (desviación estándar) 12.9 años y para los hombres fue de $39.8+14.0$ años.

Respecto al tipo de vivienda el $97.8 \%$ vivía en casa-habıtación, el 55.5\% tenía como parentesco esposo (a) y el $23.4 \%$ jefe de familia. El $49.2 \%$ se dedicaba al hogar y el 41.8 mencionaron ser trabajadores. El 48\% mencionó no recibir remuneración por su trabajo y el $23.4 \%$ eran empleados $u$ obreros.

En relación a la escolaridad para las mujeres el promedio fue de $8.6+3.7$ y para los hombres fue de $9.8+4.5$ años de escolaridad.

El $48 \%$ de la población del estudio mencionó tener antecedentes de diabetes. La rama familiar con antecedentes de diabetes con mayor porcentaje fue la materna con un $23 \%$ y el $15.6 \%$ la rama paterna. El $12.5 \%$ refirió tener diagnóstico de diabetes y el promedio de años de diagnóstico fue de $7.6+6.0$ años. El 18\% de las mujeres $(n=184)$ refirió haber tenido productos macrosómicos. Respecto a la práctica de ejercicio el 19\% de las mujeres si lo realizaba y el $29 \%$ de los hombres también $(n=72)$.

En relación a la tensión arterial sistólica el 10.6\% de la población presentó algún grado de hipertensión arterial sistólica (HTAS) y el 3.6\% algún grado de hipertensión arterial diastólica (HTAD). Es importante resaltar que en la clasificación de normal alta el $10.1 \%$ de la población presentó tensión arterial sistólica y el $8.5 \%$ tensión arterial diastólica. Al realizar las determinaciones de glucosa. capilar casual, el $81.6 \%$ obtuvo cifras $<110 \mathrm{mg} / \mathrm{dl}$, el 6.3 $\%$ de 110 a $125 \mathrm{mg} / \mathrm{dl}$, el $6.6 \%$ de 126 a $199 \mathrm{mg} / \mathrm{dl}$ y el $5.5 \%$ obtuvo cifras de $\geq 200 \mathrm{mg} / \mathrm{dl}$.

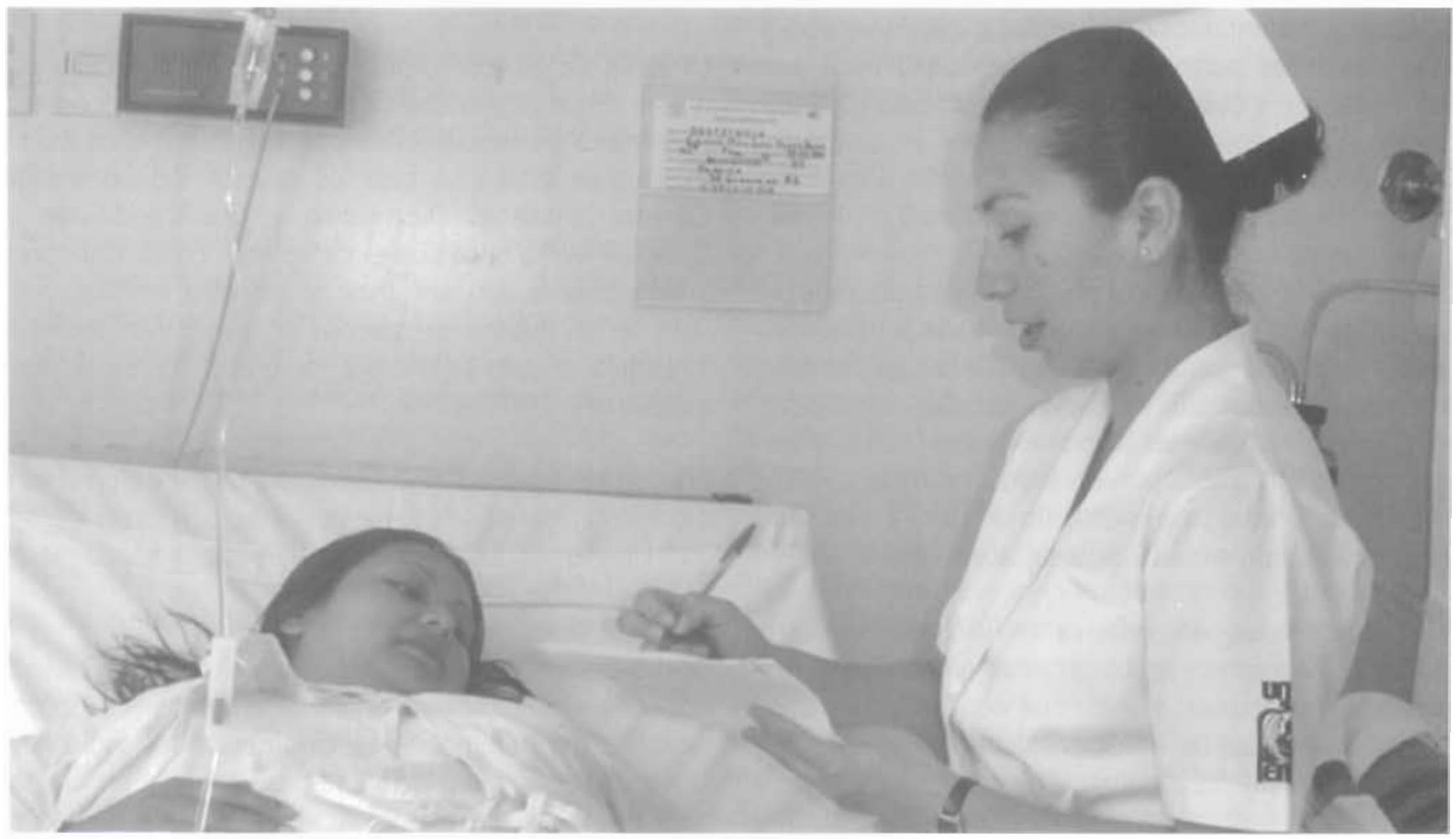


El IMC en parámetros de normalidad se presentó sólo en el $18.0 \%$ de la población de estudio. El ICC en parámetros de normalidad para el género femenino se presentó en el $31.5 \%<.84$ y el $68.5 \%>84$, para el género masculino el $52.8 \%$ fue de $<.93$ y el $47.2 \%$ se ubicó en $>.93$.

Al aplicar la prueba de X de correlación de continuidad se pudo comparar muestras independientes con un nivel de medición nominal. Al contar con antecedentes de DM por género se encontró que no hay diferencias significativas ya que la proporción de antecedentes de DM fue igual entre hombres y mujeres $\left(X^{2}=.625, g\right)=2$, prob. .269), de igual manera se mostró en relación con los antecedentes de DM ya sea en rama materna o paterna $\left(X^{2}=4.977, g \mid=4\right.$, prob. 290$)$.

En relación con la proporción de sujetos que tienen diagnóstico de DM en los distintos intervalos de edad se encontró una diferencia significativa $\left(X^{2}=29.130, \mathrm{~g}\right)=4$, prob. .000) ya que la proporción de sujetos con DM diagnosticada no fue igual en los distintos grupos de edad, 2 sujetos de cada 10 oscilaron en las edades de 40 a 59 años; asi como 3 sujetos de cada 10 dentro del grupo de 60 a 69 años.

Llama la atención con respecto a los sujetos con diagnóstico de DM en relación a su ocupación se encontró una diferencia estadisticamente significativa: $\left(X^{2}=13.228\right.$; gl $=5$, prob, 0.021) esto quiere decir que 4 personas de cada 10 que se dedican al hogar tienen diagnóstico de DM, asị como cada 2 de cada 10 que son trabajadores.

Se identificó si existía una proporción igual de sujetos que hacen ejercicio de acuerdo a los grupos se edad no se encontró una diferencia significativa $\left(X^{2}=4.105, g l=8\right.$, prob. 848). Esto demuestra que la proporción de sujetos que realizan ejercicio, no es igual en los distintos grupos de edad. Nótese que 3 de cada 10 sujetos de menos de 39 años sí lo practican y 4 de cada 10 sujetos de 60 a 69 años también lo realizan.

Al aplicar t de student: para muestras independientes y variables intervalares para identificar si era igual la proporción de sujetos hombres o mujeres que presentaron algún grado de hipertensión se obtuvo una diferencia significativa $(t=2.668, g)=123.827$, prob. .008). Por lo tanto, el nivel de HTAS no es igual entre hombres y mujeres. Los hombres tienden más a elevar su HTAS en nivel normal mientras que las mujeres se encuentran en nivel óptimo.

Al identificar si es igual la proporción de sujetos que realizan ejercicio con la presencia de algún grado de HTAD, se encontró una diferencia estadisticamente significativa ( $\mathrm{t}=$ 13.590, $g l=255$, prob. .000). El nivel de HTAD no es igual entre sujetos que practican ejercicio, ya que quienes realizan ejercicio y lo hacen regularmente, tienden a una tensión arterial óptima, mientras que quienes no lo realizan elevan sus cifras de HTAD, fuera de los parámetros de normalidad.

Al identificar si es igual la proporción de sujetos que presentaron HTAS en relación a la ocupación, se encontró una diferencia significativa $(t=14.715, \mathrm{gl}=251$, prob. .000 ). Esto indica que el nivel de HTAS no es igual en los distintos grupos de ocupación, ya que trabajadores, estudiantes y amas de casa asi como jubilados, registraron una tensión arterial que osciló de óptima a normal; mientras que los jubilados o los individuos incapacitados permanentes, presentaron una HTAS de normal alta al rango de 140 a $159 \mathrm{mmHg}$.

Al aplicar ANOVA para conocer si es igual la proporción de sujetos que presentaron HTAS en relación a la edad se identificó un diferencia estadisticamente significativa ( $F=$ 19.570), $g \mid=$ intergrupos $=4, g \mid=$ intragrupo $=247$, prob. $.000)$, esto es que la proporción de sujetos que tienen HTAS, fuera de los parámetros normales no es igual en los distintos grupos de edad, en el grupo de 50 a 59 años de edad tienen una HTAS normal alta, mientras que el grupo de 20 a 40 años tienen una presión de óptima a normal.

Al aplicar la prueba de asociación de Pearson. (n) para variạbles intervalares, șe buṣcọ la relación existente entre la HTAD en relación con la edad; se identificó $(r=+$ 0.273, prob. .000), por lo tanto existe una asociación líneal positiva leve entre la presión arterial diastólica y la edad en los individuos del presente estudio. Esto indica que a mayor edad, mayor HTAD y viceversa.

Se identificó si era igual el nivel de glucosa capilar en los distintos grupos de edad al aplicar ANOVA (ver tabla l). Se encontró que el nivel de glucosa capilar no era igual en los distintos grupos de edad. El nivel de glucosa capilar en sujetos de 20 a 39 años la media osciló entre 85 y 93. $\mathrm{mg} / \mathrm{dl}$; mientras que conforme aumentó la edad, también esta aumenta, por ejemplo en los de 50 a 59 años, el nivel de glucosa medio se encontró en $136 \mathrm{mg} / \mathrm{dl}$ y en el grupo de 60 a 69 años fue de 154 mg/dl.

Se identificó si el nivel de glucosa capilar era igual en relación a la ocupación y se encontró que el nivel de glucosa capilar no es igual en individuos de distintas ocupaciones (ver tabla II). La media de estudiantes y trabajadoras del hogar osciló entre 72 y 75 mg/dl de glucosa; mientras 
Tabla I

Nivel de glucosa capilar en los distintos grupos de edad. Prueba ANOVA

\begin{tabular}{llllll} 
& $\begin{array}{l}\text { Suma } \\
\text { de cuadrados }\end{array}$ & $\begin{array}{l}\text { Grados de } \\
\text { libertad }\end{array}$ & $\begin{array}{l}\text { Media de } \\
\text { cuadrados }\end{array}$ & F & *P \\
\hline Entre grupos & 139329.1 & 4 & 34832.275 & 10.834 & .000 \\
Con grupos & 806959.9 & 251 & 3214.980 & & \\
Total & 946289 & 255 & &
\end{tabular}

Tabla II

Nivel de glucosa capilar era igual en relación

a la ocupación. Prueba ANOVA

\begin{tabular}{llllll} 
& $\begin{array}{l}\text { Suma } \\
\text { de cuadrados }\end{array}$ & $\begin{array}{l}\text { Grados de } \\
\text { libertad }\end{array}$ & $\begin{array}{l}\text { Media de } \\
\text { cuadrados }\end{array}$ & F & *P \\
\hline Entre grupos & 180830.8 & 5 & 36166.169 & 11.812 & .000 \\
Con grupos & 765458.1 & 250 & 3061.833 & & \\
Total & 946289.0 & 255 & & &
\end{tabular}

Tabla III

Clasificación general del IMC por grupo de edad

\begin{tabular}{|c|c|c|c|c|c|}
\hline \multirow{2}{*}{$\begin{array}{c}\text { Clasificación del IMC } \\
\qquad \mathrm{kg} / \mathrm{m}^{2}\end{array}$} & \multicolumn{5}{|c|}{ Edad } \\
\hline & $\begin{array}{l}20-29 \\
\mathrm{n} / \%\end{array}$ & $\begin{array}{l}30-39 \\
\mathrm{n} / \%\end{array}$ & $\begin{array}{c}40-49 \\
n / \%\end{array}$ & $\begin{array}{c}50-59 \\
\mathrm{n} / \%\end{array}$ & $\begin{array}{c}60-69 \\
n / \%\end{array}$ \\
\hline $\begin{array}{l}\text { Normal } \\
(18.5-24.9)\end{array}$ & $\begin{array}{c}27 \\
(33.0)\end{array}$ & $\begin{array}{c}11 \\
(16.4)\end{array}$ & $\begin{array}{c}4 \\
(9.3)\end{array}$ & $\begin{array}{c}3 \\
(7.3)\end{array}$ & $\begin{array}{c}1 \\
(4.4)\end{array}$ \\
\hline $\begin{array}{l}\text { Sobrepeso } \\
(25-29.9)\end{array}$ & $\begin{array}{c}38 \\
(46.3)\end{array}$ & $\begin{array}{c}33 \\
(49.2)\end{array}$ & $\begin{array}{c}20 \\
(46.5)\end{array}$ & $\begin{array}{c}8 \\
(19.5)\end{array}$ & $\begin{array}{c}12 \\
(52.2)\end{array}$ \\
\hline $\begin{array}{l}\text { Obesidad grado I } \\
(30.0-34.9)\end{array}$ & $\begin{array}{c}12 \\
(14.6)\end{array}$ & $\begin{array}{c}16 \\
(24.0)\end{array}$ & $\begin{array}{c}12 \\
(28.0)\end{array}$ & $\begin{array}{c}17 \\
(41.5)\end{array}$ & $\begin{array}{c}4 \\
(17.4)\end{array}$ \\
\hline $\begin{array}{l}\text { Obesidad grado } \| \\
(35.0-39.9)\end{array}$ & $\begin{array}{c}4 \\
(4.9)\end{array}$ & $\begin{array}{c}7 \\
(10.4)\end{array}$ & $\begin{array}{c}4 \\
(9.3)\end{array}$ & $\begin{array}{c}10 \\
(24.4)\end{array}$ & $\begin{array}{c}6 \\
(26.0)\end{array}$ \\
\hline $\begin{array}{l}\text { Obesidad grado III } \\
(>40.0)\end{array}$ & $\begin{array}{c}1 \\
(1.2)\end{array}$ & 0 & $\begin{array}{c}3 \\
(6.9)\end{array}$ & $\begin{array}{c}3 \\
(7.3)\end{array}$ & 0 \\
\hline
\end{tabular}

Fuente: Cédula de entrevista ESVIDIM-2

que los jubilados obtuvieron una media de $157 \mathrm{mg} / \mathrm{dl}$; es decir que el nivel de glucosa capilar podría estar inversamente proporcional al nivel de actividad física dado por la ocupación.

Al identificar si era igual la proporción de sujetos obesos en los distintos grupos de edad se identificó una diferencia estadisticamente significativa $\left(F=1^{\circ} .644\right.$, g|mergupes $=4$, glintragupo $=251$, prob. .000), lo cual indica que no es igual en los distintos grupos de edad (ver tabla III).

\section{DISCUSIÓN}

El incremento de la transición epidemiológica de la diabetes mellitus tipo 2 se ve reflejada en la prevalencia ascendente. La ENSA (2000) registró una prevalencia de 
diabetes mellitus tipo 2 del $10.9 \%$ y el estudio muestra una diferencia minima del $12.5 \%$, lo cual puede deberse al incremento en la incidencia del año 2000 al año 2003 en el que se realizaron las valoraciones, sin embargo, una limitante del estudio fue no haber realizado detecciones de la glucosa capilar en ayuno.

Un aspecto coincidente con la ENSA (2000) fue que se observó un incremento drástico en la prevalencia en los grupos de edad de 50 a 59 y en el grupo de edad de 60 a 69 años. Esto indica la necesidad creciente que tienen los adultos de disminuir el consumo de carbohidratos y grasas saturadas, incremento en la actividad fisica, a fin de disminuir el riesgo para el desarrollo de la diabetes mellitus tipo 2. Por otro lado, muestra la tendencia del envejecimiento de la población y el incremento de los riesgos debidos a la urbanización y sedentarismo progresivo de la vida moderna.

Vázquez Ch. C. et al (2003), refieren en su estudio que a mayor IMC se incrementa el Indice Cintura Cadera (ICC) lo cual coincide con el estudio, sin embargo es importante señalar que en los grupos de menor edad se observan cifras de ICC más elevadas, lo cual significa un riesgo mayor para el desarrollo de diabetes mellitus tipo 2, en la población de estudio.
Es importante señalar que con respecto al IMC sólo el 18.0 $\%$ de la población en estudio se ubicó en un parámetro de normalidad y el mayor porcentaje se situó en el rubro de sobrepeso y obesidad, lo cual coincide con el estudio de (Sánchez-Reyes) y col (2001). Aunado a esto, se observa que en el género femenino solo un minimo porcentaje de la población de estudio refiere realizar ejercicio y se observa un ligero incremento en el género masculino.

La prevención de la diabetes mellitus tipo 2 se asocia con la detecciọn temprana y modificación de factores de riesgo, principalmente en la población mayor de 45 años, obesos; con un IMC y un ICC de riesgo y lá práctica de ejercicio.

\section{CONCLUSIONES}

Es notorio que un gran porcentaje de la población en estudio muestra una tendencia a la obesidad y sobrepeso con elevadas cifras de IMC e ICC. Por lo que se recomienda ampliar la promoción a la salud a través de estudios de intervención que incidan en cambios de conducta para la modificación de factores de riesgo tales como: el IMC y el ICC.

Se desconoce la incidencia anual de la diabetes mellitus tipo 2, en población abierta, por lo que se sugiere realizar detecciones anuales, asi como fomentar en la comunidad

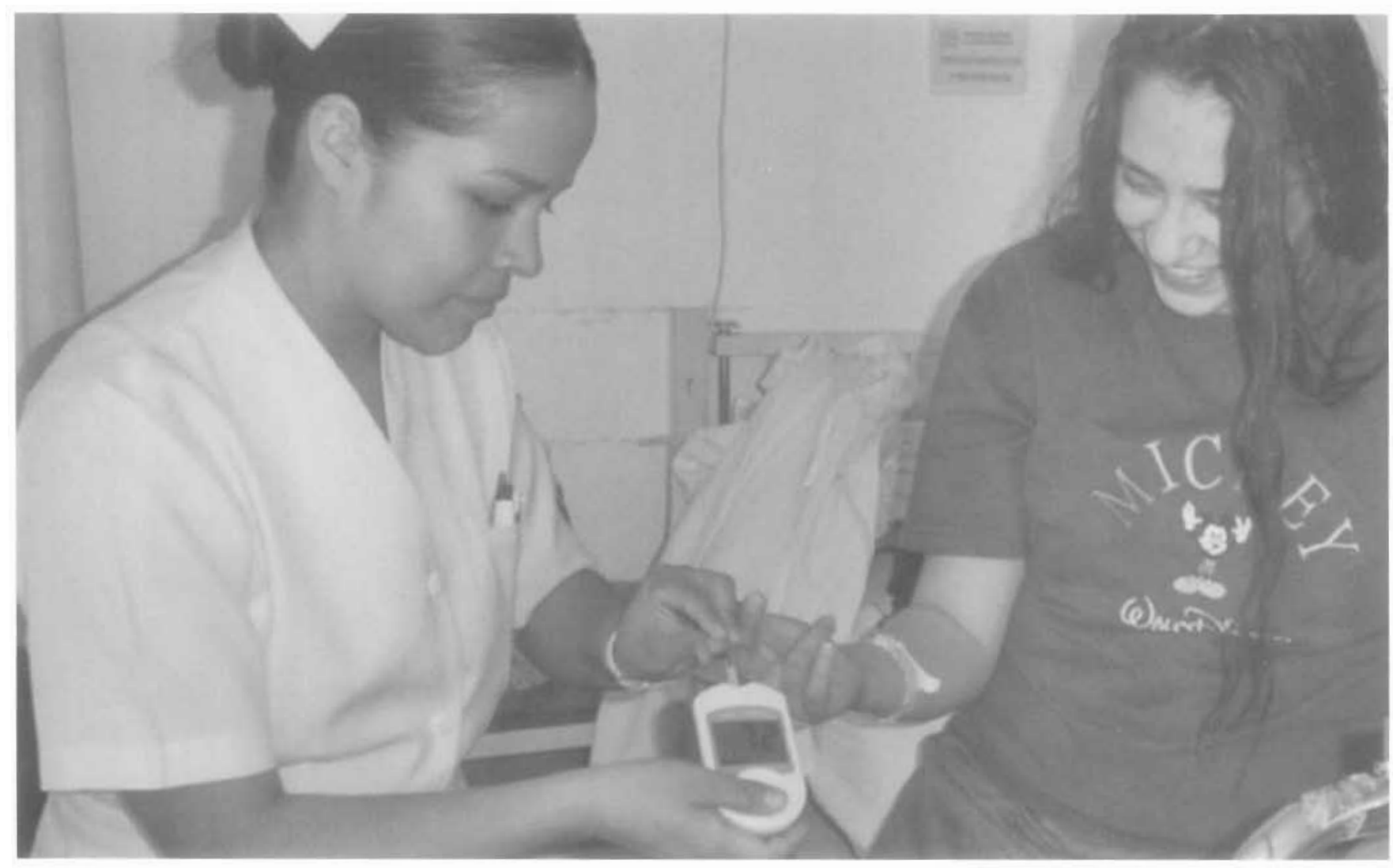


las determinaciones rutinarias de glucosa capilar en ayuno principalmente de la población mayor de 40 años.

\section{AGRADECIMIENTOS}

Agradecemos al Programa de Apoyo a Proyectos de investigación e Innovación Tecnológica (PAPIT) de la Dirección General de Asuntos del Personal Académico (DGAPA) de la UNAM por el financiamiento del programa ESVIDIM-2, y muy especialmente a todos los pasantes de servicio social que participaron en el desarrollo de esta investigación. Asi mismo, agradecemos a las personas de la comunidad en estudio y al personal de Centro Comuritario de Atención Primaria de la ENEO de la UNAM.

\section{REFERENCIAS BIBLIOGRÄFICAS}

1. Organización Panamencana de la Salud en <http://wrw.paho.org/Spanish/DPI/ps021113 hum\#\#> Septiembre 2005 p. 1 -4

2. Secretaria de Salud, Dirección Genera! de Epidemiologia, Instituto Nacional de la Nutrición Salvador Zubirán. Encuesta Nacronal de Salud', Secretaria de Salud; México, 2000. p. 94-102
3. Vázquez Ch. C. Salinas, O. S. Gómez, D. R. A., Rosso, J. Ma. M., Limènez, V. M. Arguero. S. R Factores metabólicos de riesgo Cardiovascular en población mexicana con diferente indice de mosa corporal Revista de Endocrinologla y Nutrición Vol. 11. No. 1. Enero - Marzo 2003, p. 15-21

4. Sánchez-Reyes, L., Berber, A. Y Fanghanel, G. Incidencia de obesidad en una población mexicano. Revista de Endocrinologia y Nutrición Vol. 9, No. 2, Abrit-junio 2001, p. 15-21.

5. ESVIDIM-2. Manual de procedimientos para la estandarización de procedimientos de glucosa capilar, peso, tallo, presión arterial y circunferencia de antura-cadera. Méxica, 2001.p. 25

6. The Expert Comité on the Diagnosis and Classification of Diabetes Mellitus. Follow-up Report on the Diagnosis of Diabetes Mellitus Diabetes Care, volumen 26, number ?1. noviembre 2003. p. 3160 3165

7. The Sixth Repont of the Joint National Committee on Prevention. Detection, Evaluation, and Treatment: of High Blood Pressure (JNC V1). Arch Intern Med 1997: 157:2413-2446

8. TANITA. (2001). "iPorqué es importante tomar medidas contro e exceso de grasa? Ultimate Scale. México; 2000/2001, p. 2

9. World Health Organization. Obesity. Preventing and managing the global epidemic. Report of a WHO Consultation on Obesity, Genova 1997. WHO, 3-5」 p. $1-16$.

\section{DIRECCIÓN PARA CORRESPONDENCIA}

Mtra. Verónica Flores Femández: febrero67@yahoo.com

Mtra. Liliand González Juárez: lilili24@yahoo.com.mx

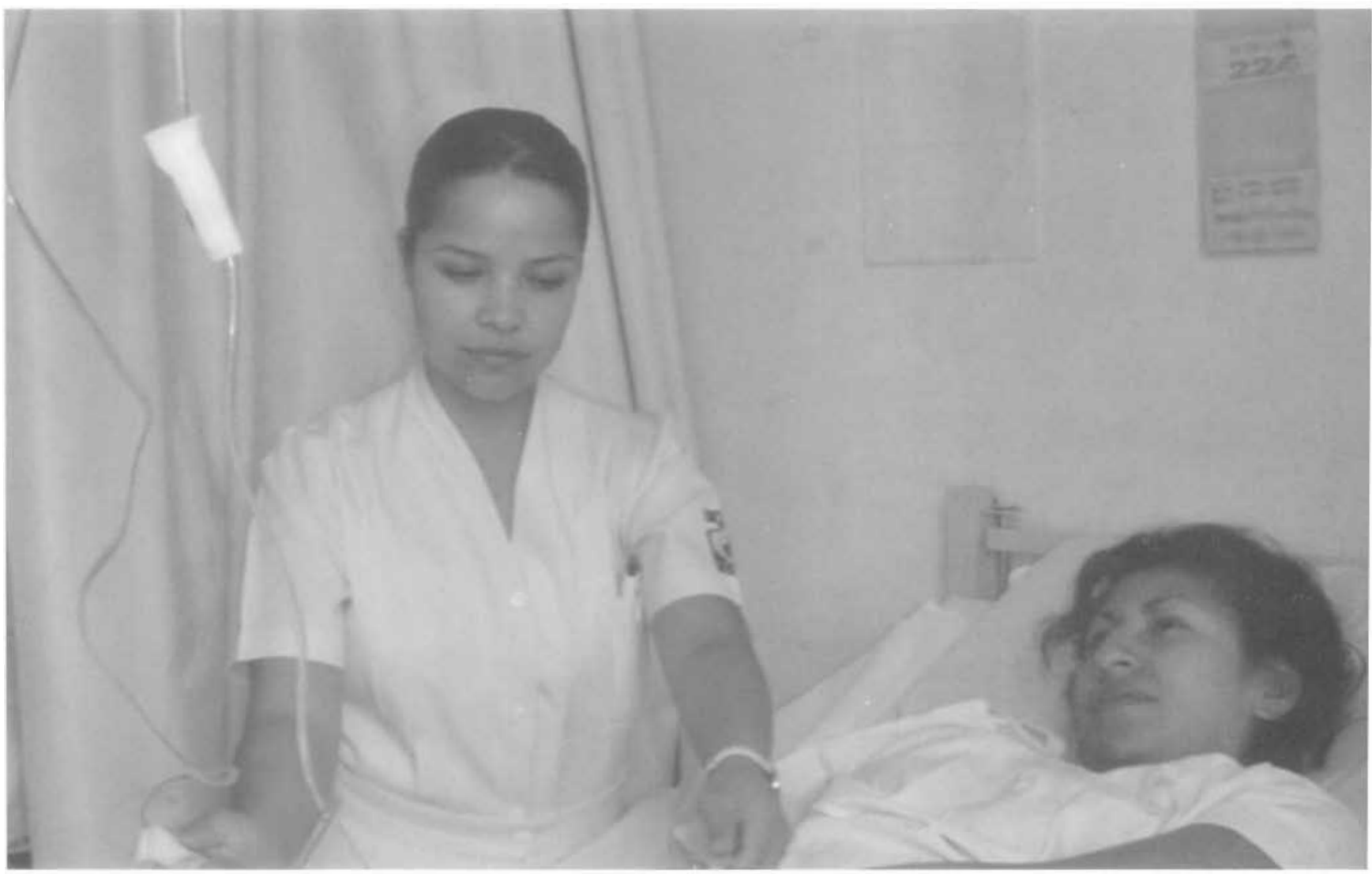

